\title{
A rapid solution adjustment strategy in solving Long-term scheduling of large cascade hydropower stations
}

\author{
Chao Wang ${ }^{1 *}$, Ji Liang $^{2 *}$, Peibing Song ${ }^{3}, \mathrm{Xin} \mathrm{Hu}^{2}$, Jiahui Sun ${ }^{2}$ \\ ${ }^{1}$ State Key Laboratory of Simulation and Regulation of Water Cycle in River Basin, China Institute of Water Resources and Hydropower \\ Research,1 Fuxing Beijing 100038, China \\ ${ }^{2}$ School of Hydropower and Information Engineering, Huazhong University of Science and Technology, Wuhan 430074, China \\ ${ }^{3}$ College of Civil Engineering and Architecture, Zhejiang University, Hangzhou 310058, China
}

\begin{abstract}
Due to the great potential of large cascade hydropower stations on power generation, long-term scheduling of large cascade hydropower stations (LSLCHS) plays an important role in electrical power system. As more and more concentrations focused on the optimal operation of large cascade hydropower stations, the LSLCHS has been taken into a multi-dimensional, non-convex and non-linear optimization problem due to its complicated hydraulic connection relationships and varieties of complex constraints with considering its power generation, shipping and ecological characteristics. In order to solve this problem, a strategy of solution rapid adjustment regarding the principle of monotone principle is proposed accordingly. The simulation results show that the proposed method is an efficient for solving joint optimization dispatch model of cascade hydropower stations with fast convergent rate, strong robustness.
\end{abstract}

\section{Introduction}

The Jinsha River is the upper stretches of the Yangtze River. Four large hydropower stations locate downstream the river, Wudongde (under construction, 8,700 MW), Baihetan (under construction, 14,000 MW), Xiluodu (completed, 13,860 MW) and XiangjiaBa (completed, $6,400 \mathrm{MW}$ ). With the gradual completion of these hydropower stations, requirements of optimal operation including long-term scheduling for power production, evolution of historical operation and compensation benefit analysis are urgent. Long-term scheduling of large cascade hydropower stations (LSLCHS) is a significance task to satisfy these needs. The objective of LSLCHS is to maximize the benefit of power production by controlling the water level processes of hydropower stations while subjecting to various constraints. In comparison to the optimization of single hydropower station, LSLCHS problem has more complex constraints due to its hydraulic and electric connection, which makes this optimization problem quite difficult to solve ${ }^{[1,2]}$.

In order to solve this problem, numerous methods have been proposed in recent years, linear programing (LP) ${ }^{[3}$, ${ }^{4]}$, none linear programming (NLP) ${ }^{[5,6]}$, dynamic programs (DP) ${ }^{[7,8]}$, discrete differential dynamic programming (DDDP) ${ }^{[9,10]}$, heuristic algorithms, such as particle swarm optimization (PSO) ${ }^{[11-13]}$, genetic algorithm $(\mathrm{GA})^{[14,15]}$, differential evolution (DE) ${ }^{[16,17]}$, cultural algorithm (CA)

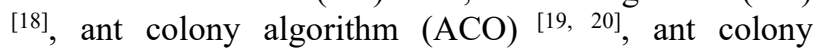
optimization for continuous domain $\left(\mathrm{ACO}_{\mathrm{R}}\right)^{[21]}$. These algorithms have been widely utilized in LSLCHS. While all these algorithms try to solving the LSLCHS by improve the algorithm mechanism, lacking consideration on the mathematical mechanism of LSLCHS. In this paper, a local search strategy based on the principle of monotone principle in LSLCHS has been proposed, which could efficiently improve the convergence rate of optimization algorithms.

The reminder of this paper is organized as follows, Section 2 introduces the formulation of LSLCHS problem as well as the problem solving strategy. In Section 3, the proposed method is applied to solve LSLCHS in Jinsha River, and the results are analysed. Finally, conclusions are summarized in Section 4.

\section{Methodology}

\subsection{Problem formulation}

The primary objective of LSLCHS problem is to gain the maximum generating benefit of large cascade hydropower stations. The objective formula and constraints are described as follows.

\subsubsection{Objective function}

The objective to maximize the benefit of power production is described as follows,

\footnotetext{
* Corresponding author. Chao Wang Tel.: +86 10 68785503; fax: +86 10 68785503. E-mail address: wangchao@iwrh.com Ji Liang Tel.: +86 27 87543992; fax: +86 25 87543992. E-mail address: larkwater1@163.com
} 


$$
B=\max \sum_{t=1}^{T} \sum_{i=1}^{N} p_{i, t} N_{i, t} \Delta t=\sum_{t=1}^{T} \sum_{i=1}^{N} p_{i, t} K_{i} Q_{i, t} H_{i, t} \Delta t
$$

$B$ represents the total benefit of power production. $T$ is amount of periods. $N$ denotes the number of hydropower stations. $N_{i, t}$ stands for output of the $i$-th hydropower station in period $t, \Delta t$ is the length of the interval, $K_{i}$ represents output coefficient which is a constant determined by the characteristic of hydropower station, $Q_{i, t}$ equals outflow through hydro-turbines and $Q_{i, t}$ is calculated by water balance equation shown in formula (2). $H_{i, t}$ is pure water head. $p_{i, t}$ is the long-term electricity price.

\subsubsection{Constraints}

(1) Water balance constraint

$$
\begin{aligned}
& V_{i, t+1}=V_{i, t}+\left(I_{i, t}-Q_{i, t}-S_{i, t}\right) \Delta t \\
& I_{i, t}=q_{i, t}+Q_{i-1, t}+S_{i-1, t}
\end{aligned}
$$

$V_{i, t}$ is reservoir storage of the $i$-th hydropower station at the beginning of period $t, I_{i, t}$ is inflow, $q_{i, t}$ stands for local inflow and $S_{i, t}$ is deserted outflow.

(3) Water level constraint

$$
\begin{aligned}
& Z_{i, t}^{\min } \leq Z_{i, t} \leq Z_{i, t}^{\max } \\
& \left|Z_{i, t}-Z_{i, t+1}\right| \leq \Delta Z_{i}
\end{aligned}
$$

$Z_{i, t}^{\min }$ and $Z_{i, t}^{\max }$ are the upper and lower water level limits and $\Delta Z_{i}$ is the maximum amplitude of water level variation.

(4) Power generating constraint

$$
N_{i, t}^{\min } \leq N_{i, t} \leq N_{i, t}^{\max }\left(H_{i, t}\right)
$$

$N_{i, t}^{\max }$ represents the maximum output. The maximum output is a function of pure water head. $N_{i, t}^{\mathrm{min}}$ is the lower output limit, which is generally called Guaranteed output.

(5) Outflow constraint

$$
Q_{i, t}^{\min } \leq Q_{i, t}+S_{i, t} \leq Q_{i, t}^{\max }
$$

$Q_{i, t}^{\max }$ is the maximum outflow limit and $Q_{i, t}^{\min }$ is the minimum outflow limit.

(6) Boundary condition

$$
\begin{aligned}
& Z_{i, 1}=Z_{i}^{\text {begin }}, Z_{i, T}=Z_{i}^{\text {end }} \\
& Z_{i}^{\text {begin }} \text { is the initial water level and } Z_{i}^{\text {end }} \text { is the }
\end{aligned}
$$
terminal water level of the $i$-th hydropower station.

\subsection{Problem Solving}

\subsubsection{A rapid solution adjustment Strategy}

A rapid solution adjustment strategy based on the principle of monotone principle of joint operation of cascade hydropower stations has been proposed. The proposed method adjusts the solution from time period 0 to $T$ station by station. Details are shown as following,
(1) The solution adjustment strategy in one step

The solution adjustment strategy in one step is aimed to adjust the solution in period $t$ of hydropower station $m$, which could improve the total power generation of cascade hydropower stations. The adjustment contains two conditions,

Condition 1: No abandon water

a. Calculate the decision space of adjustment outflow in period $t$ of hydropower station $m$

The adjustment outflow $\Delta Q_{\mathrm{t}}$ should satisfy constraints of the hydropower stations. The decision space $\left[\Delta Q_{t}^{\min }, \Delta Q_{t}^{\max }\right]$ of outflow can be calculated by formula (9).

$$
\begin{aligned}
& Q_{t, \text { min }}^{j} \leq Q_{t}^{j}+\Delta Q_{t} \leq Q_{t, \text { max }}^{j} \\
& Q_{t+1, \text { min }}^{j} \leq Q_{t+1}^{j}-\frac{\Delta T_{t}}{\Delta T_{t+1}} \Delta Q_{t} \leq Q_{t+1, \text { max }}^{j} \\
& Z_{t+1, \text { min }}^{j} \leq Z\left(V_{t+1}^{j}-\Delta Q_{t} \Delta T_{t}\right) \leq Z_{t+1, \text { max }}^{j}
\end{aligned}
$$

$Q_{t, \max }^{\prime}$ and $Q_{t, \text { min }}^{\prime}$ are the maximum and minimum outflow of hydropower station $m$ in period $t, Z_{t+1, \text { min }}^{j}$ and $Z_{t+1, \text { max }}^{j}$ are the maximum and minimum water level of hydropower station $m$ at the beginning of period $t$.

b. Calculate the best adjustment outflow

The best adjustment outflow may be the boundary of the decision space or the value which make $\partial \Delta E_{\text {total }} / \partial \Delta Q_{t}=0 \cdot \Delta E_{\text {total }}$ is the increment of power generation caused by outflow adjustment.

b1. If $\Delta E_{\text {total }}{ }^{\prime}\left(\Delta Q_{t}^{\text {min }}\right)<0$, the best adjustment outflow take $\Delta Q_{t}^{\min }$;

b2. If $\Delta E_{\text {total }}{ }^{\prime}\left(\Delta Q_{t}^{\text {min }}\right)<0$, the best adjustment outflow take $\Delta Q_{t}^{\max }$;

b3. If $\Delta E_{\text {total }}{ }^{\prime}\left(\Delta Q_{t}^{\text {min }}\right)>0, \Delta E_{\text {total }}{ }^{\prime}\left(\Delta Q_{t}^{\text {max }}\right)<0$, the best adjustment outflow take the value which make $\partial \Delta E_{\text {total }} / \partial \Delta Q_{t}=0$.

Condition 2: Exist abandon water

If the abandon water is existing, output calculation function is continuous and non-differentiable. In this condition, $\partial \Delta E_{\text {total }} / \partial \Delta Q_{t}$ is non-existent. So we used the following strategy to get the adjust outflow.

a. Calculate the maximum abandon water

Calculate the maximum abandon water $\Delta Q_{t}^{\text {abandon }}$ in period $t$ and $t+1$ by formula (10);

$$
\Delta Q_{t}^{\text {abandon }}=\max \left(Q_{t}^{j}-Q_{t, \max }^{j}\right), j \in \mathrm{M}
$$

b. Calculate the best adjustment outflow

The best adjustment outflow can be calculated by the following steps,

b1. If $\Delta Q_{t}^{\text {abandon }}<0$, the best adjustment outflow take $\Delta Q_{t}^{\text {abandon }}$;

b2. If $\Delta Q_{t+1}^{\text {abandon }}<0$, the best adjustment outflow take $\frac{\Delta T_{t+1}}{\Delta T_{t}} \Delta Q_{t+1}^{\text {abandon }}$

(2) The process of solution adjustment

a. Parameter initialization: set $t=0, i=0$, $t \in\{0,1, \ldots, T-1\}, i \in\{0,1, \ldots, M\}, T$ is the amount of time periods and $M$ is the amount of hydropower stations;

b. Using the solution adjustment strategy in one step to adjust the solution of the $i$-th hydropower station in period $t$, and then set $t^{++}$, turn to step c; 
c. If $i>M$, turn to step d; Otherwise, if $t>T-1$, set $t$ $=0, i++$, turn to steps $\mathrm{b}$;

d. The end.

\subsubsection{Algorithms}

In order to prove the performance of the proposed method, ant colony optimization for continuous domain $\left(\mathrm{ACO}_{\mathrm{R}}\right)^{[22]}$ is adopt as the optimization algorithm. The rapid solution adjustment strategy was used as daemon actions in $\mathrm{ACO}_{\mathrm{R}}$. The framework of $\mathrm{ACO}_{\mathrm{R}}$ contains three parts, solution construction, pheromone update and daemon actions..

(1) Solution Construction

A Gaussian kernel function, which is made up of a series of Gaussian functions, is used as the probability

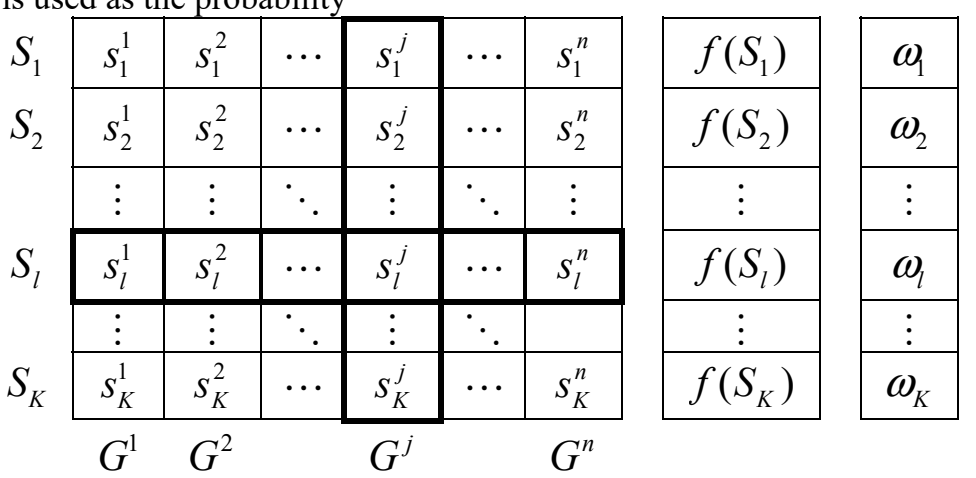

Fig.1. Solution archive $T$ with $K$ solutions.

Each row represents a solution $S_{l}$ and records its dimension value $S_{l}^{j}$, fitness value $f\left(S_{l}\right)$ and weight $\omega_{l}$. For each dimension, there is an individual Gaussian kernel PDF $G^{\mathrm{i}}$. The weight parameter $\omega_{l}$ is calculated by formula (12),

$$
\omega_{l}=\frac{1}{q K \sqrt{2 \pi}} e^{-\frac{(l-1)^{2}}{2 q^{2} K^{2}}}
$$

where $K$ is the size of $T$ and $q$ is a control parameter.

$M$ new solutions will be constructed by Gaussian kernel PDFs at each generation time. A new solution is constructed from dimension 1 to $n$. In each dimension, an individual Gaussian function $g_{l}^{i}(x)$ will be chosen by formula (13) and the sampling value of $g_{l}^{i}(x)$ is used as the solution value.

$$
p_{l}=\frac{\omega_{l}}{\sum_{r=1}^{K} \omega_{r}}
$$

$g_{l}^{i}(x)$ contains two parameters, $\sigma$ and $\mu$. In archive $T, \mu_{l}^{i}=s_{l}^{i}$ and $\sigma_{l}^{i}$ is calculated by formula (14), $\xi$ is a parameter affecting the speed of convergence.

$$
\sigma_{l}^{i}=\xi \sum_{e=1}^{k} \frac{\left|s_{e}^{i}-s_{l}^{i}\right|}{K-1}
$$

(2) Pheromone Update

The pheromone update is executed by adding new solutions to $T$ and removing the same number of worst density function (PDF) to generate solutions.

$$
G^{i}(x)=\sum_{l=1}^{K} \omega_{l} g_{l}^{i}(x)=\sum_{l=1}^{K} \omega_{l} \frac{1}{\sigma_{l}^{i} \sqrt{2 \pi}} e^{-\frac{\left(x-\mu_{l}^{i}\right)^{2}}{2 \sigma_{l}^{i}}}
$$

The Gaussian kernel function is decided by three parameters, $\omega$ is the weight of individual Gaussian function, $\mu$ is the sample value, $\sigma$ is the standard deviation. $K$ is the size of Gaussian kernel function. One Gaussian kernel function is made up of $K$ individual Gaussian functions.

Another basic conception that must be mentioned in advance is the pheromone. In $\mathrm{ACO}_{\mathrm{R}}$, a solution archive ranked by fitness value is adopted to be the pheromone of the ant colony (shown in Fig. 1). solutions out.

(3) Daemon Actions

Daemon action is a measure to improve the quality of solution, such as local search. As a basic algorithm, $\mathrm{ACO}_{\mathrm{R}}$ takes no daemon action to improve the performance.

\section{Case Study}

In order to prove the performance of the proposed method, a case study about LSLCHS problem downstream Jinsha river has been conducted. The rapid solution adjustment strategy was used as daemon actions in $\mathrm{ACO}_{\mathrm{R}}$, which called improved $\mathrm{ACO}_{\mathrm{R}}\left(\mathrm{IACO}_{\mathrm{R}}\right)$. Cases are named after the year of the historical daily runoff, "1989" as the wet year case, "1958" as the dry year case and "2009" as the normal year case. Simulation of this problem is based upon a Java implementation running on a personal computer, Winds7 Ultimate server Pack1 x64, Intel(R) Core(TM) i5-2320 CPU@ 3.00GHZ, RAM 4.00GB. Comparison results in different inflow conditions are shown in Table1. From Table1, it is obvious that the performance of $\mathrm{IACO}_{\mathrm{R}}$ is better than $\mathrm{ACO}_{\mathrm{R}}$ in both inflow conditions. The gained maximum power generation by $\mathrm{IACO}_{\mathrm{R}}$ is 1.01 billion, 0.45 billion and 0.28 billion more than which gained by $\mathrm{ACO}_{\mathrm{R}}$ in wet year, normal year and dry year, respectively. On the convergence precision aspect, the performance of $\mathrm{IACO}_{\mathrm{R}}$ is better than $\mathrm{ACO}_{\mathrm{R}}$, shown in Fig.2. In general, the rapid solution adjustment strategy has effectively improve the performance of $\mathrm{ACO}_{\mathrm{R}}$, and it is an optional tool to solve LSLCHS problem accurately and fleetly. 
Table1. The best solution gained by $\mathrm{ACO}_{\mathrm{R}}$ and $\mathrm{IACO}_{\mathrm{R}}$ in 100 independent simulations (billion $\mathrm{kWh}$ )

\begin{tabular}{|c|c|c|c|c|c|c|c|c|c|}
\hline \multirow{2}{*}{ Methods } & \multicolumn{3}{|c|}{ Wet year } & \multicolumn{3}{|c|}{ Normal year } & \multicolumn{3}{|c|}{ Dry year } \\
\hline & Max & Mean & Std. & Max & Mean & Std. & Max & Mean & Std. \\
\hline $\mathrm{ACO}_{\mathrm{R}}$ & 230.76 & 229.98 & 4.91 & 211.59 & 211.35 & 0.13 & 197.28 & 197.07 & 0.08 \\
\hline $\mathrm{IACO}_{\mathrm{R}}$ & 231.10 & 230.99 & 0.35 & 211.86 & 211.80 & 0.02 & 197.39 & 197.34 & 0.02 \\
\hline Difference & 0.34 & 1.0.1 & -0.46 & 0.27 & 0.45 & -0.11 & 0.11 & 0.28 & -0.06 \\
\hline
\end{tabular}

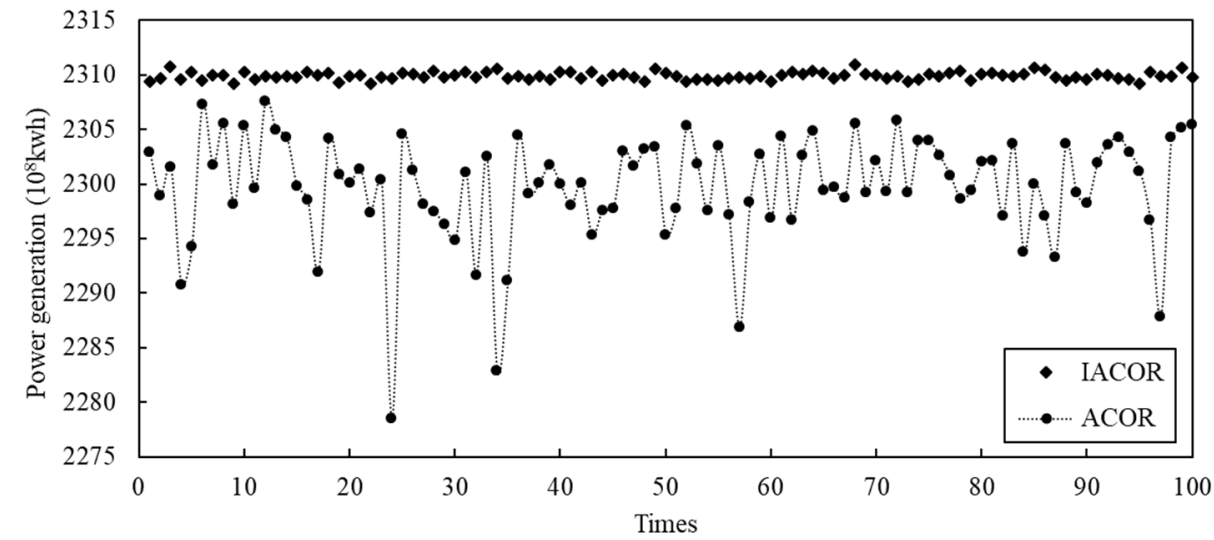

Fig.2 The maximum power generation gained by ACOR and IACOR in 100 independent simulations

\section{Conclusion}

In this paper, a rapid solution adjustment strategy is proposed to solve long-term scheduling of large cascade hydropower stations problem. To verify its performance, a case study about LSLCHS problem downstream Jinsha river has been conducted. Simulation results show that the proposed rapid solution adjustment strategy has effectively improve the performance of the original algorithm, which means that it is an effectively enhancement tool for optimization algorithms in solving LSLCHS.

\section{References}

1. M. Mahmoud, K. Dutton, M. Denman. Dynamical modelling and simulation of a cascaded reserevoirs hydropower plant. Electric Power Systems Research. 70 (2004) 129-39.

2. M. Cârdu, T. Bara. Romanian achievement in hydropower plants. Energy Conversion and Management. 39 (1998) 1193-201.

3. J.S. Windsor. Optimization model for the operation of flood control systems. Water Resources Research. 9 (1973) 1219-26.

4. J.-H. Yoo. Maximization of hydropower generation through the application of a linear programming model. Journal of hydrology. 376 (2009) 182-7.

5. J.L.B. Brandão. Performance of the equivalent reservoir modelling technique for multi-reservoir hydropower systems. Water resources management. 24 (2010) 3101-14.

6. J. Catalão, H. Pousinho, V. Mendes. Scheduling of head-dependent cascaded hydro systems: Mixedinteger quadratic programming approach. Energy Conversion and Management. 51 (2010) 524-30.
7. R.R. Shoults, R.K. Chakravarty, R. Lowther. Quasistatic economic dispatch using dynamic programming with an improved zoom feature. Electric power systems research. 39 (1996) 215-22.

8. D.N. Kumar, F. Baliarsingh. Folded dynamic programming for optimal operation of multireservoir system. Water Resources Management. 17 (2003) 337-53.

9. V. Te Chow, G. Cortes-Rivera, A. No. Application of DDDP in water resources planning. University of Illinois at Urbana-Champaign, Water Resources Center1974.

10. M. Heidari, V.T. Chow, P.V. Kokotović, D.D. Meredith. Discrete differential dynamic programing approach to water resources systems optimization. Water Resources Research. 7 (1971) 273-82.

11. J. Chuanwen, E. Bompard. A self-adaptive chaotic particle swarm algorithm for short term hydroelectric system scheduling in deregulated environment. Energy Conversion and Management. 46 (2005) 2689-96.

12. C.-t. Cheng, S.-1. Liao, Z.-T. Tang, M.-y. Zhao. Comparison of particle swarm optimization and dynamic programming for large scale hydro unit load dispatch. Energy Conversion and Management. 50 (2009) 3007-14.

13. R. Zhang, J. Zhou, Y. Lu, H. Qin, H. Zhang. A PSObased bacterial chemotaxis algorithm and its application. Advances in Neural Networks-ISNN 2011. Springer2011. pp. 219-27.

14. R. Wardlaw, M. Sharif. Evaluation of genetic algorithms for optimal reservoir system operation. Journal of Water Resources Planning and Management. 125 (1999) 25-33.

15. C.-T. Cheng, W.-C. Wang, D.-M. Xu, K. Chau. Optimizing hydropower reservoir operation using 
hybrid genetic algorithm and chaos. Water Resources Management. 22 (2008) 895-909.

16. Y. Lu, J. Zhou, H. Qin, Y. Li, Y. Zhang. An adaptive hybrid differential evolution algorithm for dynamic economic dispatch with valve-point effects. Expert Systems with Applications. 37 (2010) 4842-9.

17. X. Yuan, B. Cao, B. Yang, Y. Yuan. Hydrothermal scheduling using chaotic hybrid differential evolution. Energy Conversion and Management. 49 (2008) 3627-33.

18. X. Yuan, Y. Yuan. Application of cultural algorithm to generation scheduling of hydrothermal systems. Energy Conversion and Management. 47 (2006) 2192-201.

19. J. Cai, X. Ma, L. Li, Y. Yang, H. Peng, X. Wang. Chaotic ant swarm optimization to economic dispatch. Electric Power Systems Research. 77 (2007) 1373-80.

20. L. Mo, P. Lu, C. Wang, J. Zhou. Short-term hydro generation scheduling of Three Gorges-Gezhouba cascaded hydropower plants using hybrid MACSADE approach. Energy Conversion and Management. 76 (2013) 260-73.

21. A. Fetanat, G. Shafipour. Generation maintenance scheduling in power systems using ant colony optimization for continuous domains based 0-1 integer programming. Expert Systems with Applications. 38 (2011) 9729-35.

22. K. Socha, M. Dorigo. Ant colony optimization for continuous domains. European Journal of Operational Research. 185 (2008) 1155-73. 